ARTICLE OPEN

\title{
How dopants limit the ultrahigh thermal conductivity of boron arsenide: a first principles study
}

Mauro Fava ${ }^{1}$, Nakib Haider Protik $\mathbb{D}^{2}$, Chunhua $\mathrm{Li}^{3}$, Navaneetha Krishnan Ravichandran ${ }^{4}$, Jesús Carrete $\mathbb{( \mathbb { D }}^{5}$, Ambroise van Roekeghem ${ }^{6}$, Georg K. H. Madsen $\mathbb{1}^{5}$, Natalio Mingo ${ }^{6}$ and David Broido $\mathbb{1}^{3 凶}$

The promise enabled by boron arsenide's (BAs) high thermal conductivity $(\kappa)$ in power electronics cannot be assessed without taking into account the reduction incurred when doping the material. Using first principles calculations, we determine the $\kappa$ reduction induced by different group IV impurities in BAs as a function of concentration and charge state. We unveil a general trend, where neutral impurities scatter phonons more strongly than the charged ones. $C_{B}$ and $G_{A s}$ impurities show by far the weakest phonon scattering and retain BAs $k$ values of over $\sim 1000 \mathrm{~W} \cdot \mathrm{K}^{-1} \cdot \mathrm{m}^{-1}$ even at high densities. Both Si and Ge achieve large hole concentrations while maintaining high $\kappa$. Furthermore, going beyond the doping compensation threshold associated to Fermi level pinning triggers observable changes in the thermal conductivity. This informs design considerations on the doping of BAs, and it also suggests a direct way to determine the onset of compensation doping in experimental samples.

npj Computational Materials (2021)7:54; https://doi.org/10.1038/s41524-021-00519-3

\section{INTRODUCTION}

Increased power densities in electronic devices lead to heightened efficiency and durability issues due to overheating, prompting the search for alternative, higher thermal conductivity $(\kappa)$ materials. Thus, the recently demonstrated high $\kappa$ of cubic boron arsenide (BAs) offers great promise, particularly for power electronics. Measured values of $\sim 1300 \mathrm{~W} \cdot \mathrm{K}^{-1} \cdot \mathrm{m}^{-1}$ have been attained at room temperature for ultra-pure samples $s^{1-3}$, in agreement with results from first principles calculations ${ }^{3-5}$. These high values can be explained in terms of a unique collection of properties including high bond stiffness and large mass ratio between As and B atoms, in a way that reduces the scattering phase space for the phonon modes in the material. It has also been predicted that BAs should have simultaneously high room temperature electron and hole mobilities of over $1000 \mathrm{~cm}^{2} \cdot \mathrm{v}^{-1} \cdot \mathrm{s}^{-16}$. This suggests the possibility that BAs could be used as an effective functional material in nextgeneration electronic devices, and its much higher thermal conductivity would minimize device failure from hot spot formation. Such a benefit is of particular importance as device sizes are shrinking and the resulting higher generated power densities are becoming an increasingly serious challenge. However, its use as such a functional material requires doping it, which could potentially destroy its advantageously large $\kappa$. Defects such as antisites, vacancies and impurities can lower the intrinsically high thermal conductivity of BAs by orders of magnitude, and nand $p$-type dopants need to be identified that preferably maintain the highest possible thermal conductivity.

In past works the role played by intrinsic point defects has been highlighted $^{7-9}$, nevertheless at the present date there is no comprehensive study about the effect of external substitution atoms. Recent investigations from first principles have addressed the thermodynamic stability of many neutral and charged dopants in $B A s^{10,11}$. Among those, the ones in column IV are of great interest due to their position between the columns of $B$ and $A s$ in the periodic table. In particular, the high p-dopability of BAs has been recently studied ${ }^{11,12}$. Photoluminescence and electron paramagnetic resonance experiments have been used ${ }^{11}$ along with ab initio calculations to point out the possibility that dopants like $\mathrm{C}$ and $\mathrm{Si}$, behaving as acceptors, might affect the BAsconductivity by virtue of their unintentional presence in boron precursor powders and boride based compounds.

Here we show how each dopant in group IV (C, Si, and $\mathrm{Ge})$, in its neutral and charged forms, affects the thermal conductivity of BAs. This unveils a general trend, where neutral impurities reduce the thermal conductivity more strongly than charged ones. We offer an interpretation in terms of the change in orbital occupation between the original and substituted system. We also highlight the initially counter-intuitive fact that, even for substitutions involving a large mass-difference value, the mass-difference scattering can be small. Finally, we show that in BAs excessive doping beyond the Fermi level pinning point activates phonon-donor scattering events, which can either slow down the decrease of thermal conductivity or cause it to plummet, depending on the type of impurity. This should be considered in future applications of BAs. Remarkably, we find that phonon scattering by $C_{B}$ and $\mathrm{Ge}_{A s}$ impurities is exceptionally weak. As a result, ultrahigh $B A s K$ values can be achieved even for high $C_{B}$ and $\mathrm{Ge}_{\mathrm{As}}$ densities. Furthermore, we predict that $\mathrm{Si}_{\mathrm{As}}$ and $\mathrm{Ge}_{\mathrm{As}}$ impurities will be useful p-type dopants with both achieving relatively high free hole densities while maintaining BAs $K$ values far higher than those in any common semiconductor.

\section{RESULTS \\ Background and theoretical approach}

In insulators and semiconductors, phonons are the major carriers of heat. An applied temperature gradient drives a phonon current. Under steady state conditions, this phonon drift is balanced by resistance from phonon scattering processes. Intrinsic thermal resistance comes from anharmonic phonon-phonon scattering ${ }^{13}$. In BAs, both lowest-order three-phonon and higher-order

\footnotetext{
${ }^{1}$ Université Grenoble Alpes, Saint-Martin-d'Hères, France. ${ }^{2}$ John A. Paulson School of Engineering and Applied Sciences, Harvard University, Cambridge, MA, USA. ${ }^{3}$ Department of Physics, Boston College, Chestnut Hill, MA, USA. ${ }^{4}$ Department of Mechanical Engineering, Indian Institute of Science, Bangalore, India. ${ }^{5}$ Institute of Materials Chemistry, TU Wien, Vienna, Austria. ${ }^{6}$ CEA, LITEN, Grenoble, France. ${ }^{凶}$ email: broido@bc.edu
} 


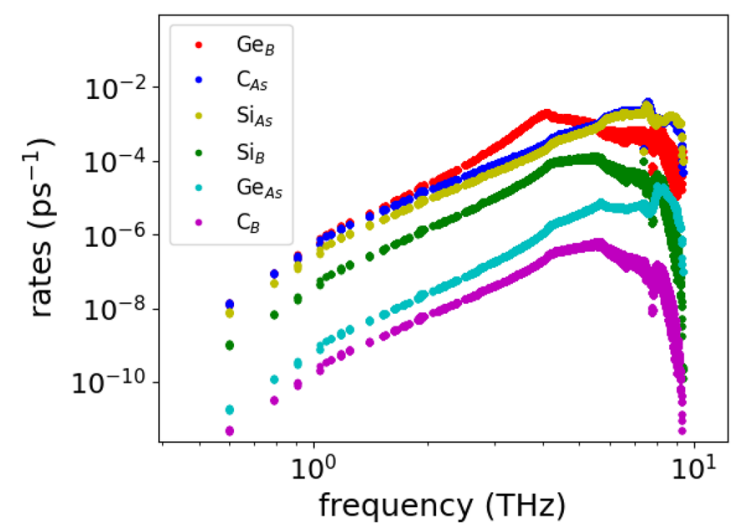

Fig. 1 Phonon-mass-defect scattering. Phonon-impurity scattering rates vs phonon frequency, including only the mass defect contribution. The doping concentration is fixed at $3.26 \cdot 10^{18} \mathrm{~cm}^{-3}$.

four-phonon scattering are required to accurately describe the intrinsic, defect-free thermal conductivity ${ }^{5}$. Substitutional dopants introduce two types of perturbations: on-site mass perturbations, $V_{M}$, resulting from the mass difference between the host and substituting atoms, and extended bond perturbations, $V_{k}$, resulting from the distortion in the local bonding environment produced by the impurity. The total perturbation, $V=V_{M}+V_{K}$, produces additional phonon scattering that lowers the BAs thermal conductivity. We assume that the dopants are randomly distributed throughout the BAs crystal and that their concentrations are sufficiently low that each defect can be treated as an independent scattering center. To describe phonon thermal transport in BAs in the presence of intrinsic three- and fourphonon scattering and C, Si and Ge dopants, we solve the phonon Boltzmann Transport Equation (BTE) ${ }^{14,15}$. Phonon-defect scattering is described using a T-matrix approach ${ }^{16,17}$, which treats the defects to all orders in perturbation theory. The theoretical approach for constructing $V_{M}$ and $V_{K}$, determining the phonon scattering rates and calculating the BAs thermal conductivity is described in detail in the Methods section.

Phonon transport in BAs is peculiar in the sense that it is dominated by phonons in a narrow window of frequencies between 4 and $8 \mathrm{THz}$. This is due to several features in BAs including a large frequency gap between acoustic and optic phonons, a narrow optic phonon bandwidth, a bunching together of acoustic phonon branches and exceptionally weak four-phonon scattering. This combination of features gives rise to unusually large contributions to $K$ from acoustic phonons in this particular range $\mathrm{e}^{3,4}$.

\section{Mass defect scattering}

Let us first look at the scattering produced by the mass difference between the introduced dopant atom and the host atom (either B or As), conveyed through the perturbation, $V_{\mathrm{M}}$. The absolute mass difference normalized by the host atom mass is $0.1(0.8)$ for $C$ substituted for $B(A s), 1.6(0.6)$ for Si substituted for $B(A s)$, and 5.7 (0.03) for $\mathrm{Ge}$ substituted for $B(A s)$. $G_{A s}$ and $C_{B}$ substitutions correspond to the smallest mass differences, since Ge and $A s$ and $\mathrm{C}$ and $\mathrm{B}$ are adjacent on the periodic table. For the rest of the substitutions the mass difference is large.

In BAs, acoustic phonons carry almost all the heat. Figure 1 shows the calculated scattering rates of acoustic phonons for impurity density of $3.26 \cdot 10^{18} \mathrm{~cm}^{-3}$ including only the mass defect perturbation $V_{\mathrm{M}}$. In contrast with the case of single-species compounds, the magnitudes of the mass defect scattering rates in Fig. 1 do not follow a monotonic behavior with respect to the normalized mass difference: in binary compounds with a large heavy-to-light mass ratio of the constituent atoms, like BAs, almost all acoustic phonon modes throughout the Brillouin zone involve dominant motion of the heavy (As) atoms, while the light (B) atoms remain relatively stationary. As a result, mass defects placed on the heavy atom sites lead to strong scattering of acoustic phonons while those placed on the light atom site become almost invisible to acoustic phonons and provide only weak scattering ${ }^{18}$. An approximated analytical expression for the mass-difference scattering rate in large mass-ratio binary compounds was given by Lindsay et al. ${ }^{18}$, and experimentally verified by Chen et al. ${ }^{19}$.

\section{Bond defect scattering}

The scattering rates for acoustic phonons including only the bond distortions, $V_{K}$, produced by neutral and charged dopants are presented in Supplementary Fig. 1. Singly charged states are assumed so that the number of valence electrons at the impurity site matches the number on the replaced host atom. Organized according to lowest formation energy ${ }^{10,11}$, these are: $\mathrm{Si}_{\mathrm{As}}^{-1}, \mathrm{Ge}_{\mathrm{As}}^{-1}$, $C_{A s}^{-1}, S_{B}^{+1}, G_{B}^{+1}, C_{B}^{+1}$. The plots reveal a remarkable trend: phonon scattering by neutral defects is generally stronger than that by charged defects. We can possibly attribute this general phenomenon to the fact that the ionized charged states more closely resemble the electronic structure of the original host: when an atom in column IV replaces an As (B) atom, it tends to get charged by accepting (donating) an extra electron, thus becoming isoelectronic with the original atom for which it has substituted. If the impurity remains neutral, however, the extra hole (electron) present at the defect site is responsible for bond perturbations on the crystal structure that do not take place when dealing with ionized states. This hypothesis deserves further investigation in the future. The effect is most noticeable in $\mathrm{Ge}_{A s}, \mathrm{C}_{B}$ and $\mathrm{Si}_{\mathrm{As}}$, as seen in Supplementary Fig. 1. These three impurities also have weaker bond defect scattering than do $\mathrm{Ge}_{\mathrm{B}}, \mathrm{Si}_{\mathrm{B}}$ and $\mathrm{C}_{\mathrm{As}}$.

To compactly express this finding, it is useful to define and evaluate a descriptor for both charged and neutral impurity states. The descriptor, $D_{\text {def; }}$, sums the phonon-bond defect scattering rates throughout the Brillouin zone which fall in the frequency range of maximum contributions to the defect-free BAs $\kappa$ :

$D_{\text {def; } K} \equiv \frac{1}{N} \sum_{\lambda} \tau_{\lambda ; K}^{-1}\left[\theta\left(\omega_{\lambda}-\omega_{1}\right)-\theta\left(\omega_{\lambda}-\omega_{2}\right)\right]$,

Here, $\tau_{\lambda: K}^{-1}$ is the phonon-defect scattering rate evaluated by including the $V_{\mathrm{K}}$ perturbation only, lambda designates a phonon mode (wave-vector and polarization), $N$ is the number of points in the reciprocal space grid, $\theta$ is the Heaviside step-function, and $\omega_{1}$ and $\omega_{2}$ are 4 and $8 \mathrm{THz}$, respectively. In Eq. (1) we consider an impurity concentration of one defect per unit cell, as using a more dilute value would only scale $D_{\text {def; }}$ by a $x$ factor. The evaluated descriptor is shown in Fig. 2 for dopants in the neutral and charged states. The latter correspond to those with the lowest formation energy $^{10,11}: \mathrm{Si}_{\mathrm{As}}^{-1}, \mathrm{Ge}_{\mathrm{As}}^{-1}, \mathrm{C}_{\mathrm{As}}^{-1}, \mathrm{Si}_{\mathrm{B}}^{+1}, \mathrm{Ge}_{\mathrm{B}}^{+1}$ and $\mathrm{C}_{\mathrm{B}}^{+1}$. Figure 2 confirms the findings from the scattering rates calculations.

\section{Phonon thermal conductivity}

Figure 3 shows plots of the BAs thermal conductivity at room temperature $(300 \mathrm{~K})$ as a function of doping concentration for each of the six impurities considered. Solid lines correspond to the neutral impurity cases, while dotted lines are for the charged defects. At low concentrations, all curves merge to the pristine thermal conductivity value of around $k=1200 \mathrm{~W} \cdot \mathrm{m}^{-1} \cdot \mathrm{K}^{-1}$, where only three- and four-phonon scattering occurs ${ }^{3-5}$. With increasing impurity concentration, the behavior of the thermal conductivity clearly reflects the behavior of the phonon-defect scattering rates: at a given dopant density, the reduction of $\kappa$ is larger for impurities in their neutral states compared with those in their charged states. Colored horizontal arrows in Fig. 3 indicate the differences between charged and neutral impurity concentrations that produce a $50 \%$ reduction in the BAs $\kappa$. The consistently larger 


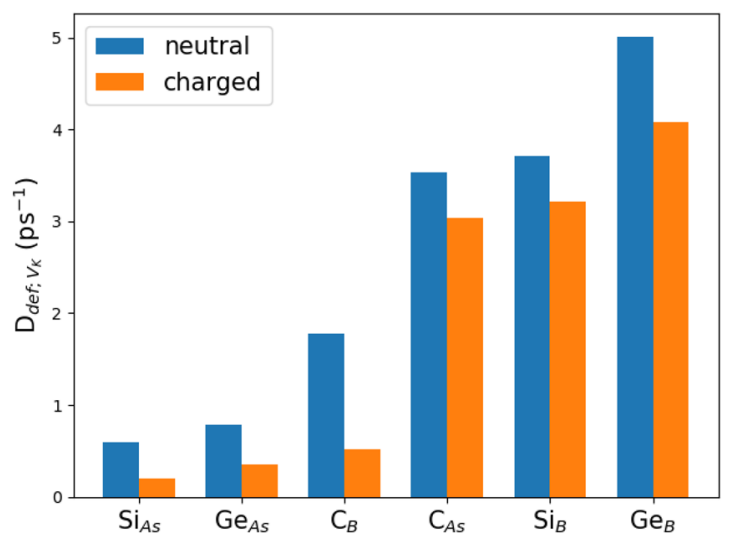

Fig. 2 Descriptor for strength of phonon-bond-defect scattering.

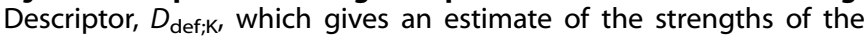
phonon-bond defect scattering rates in BAs from charged and neutral $\mathrm{Si}_{\mathrm{A}}, \mathrm{Ge}_{\mathrm{As}}, \mathrm{C}_{\mathrm{B}}, \mathrm{C}_{\mathrm{As}}, \mathrm{Si}_{\mathrm{B}}$, and $\mathrm{Ge}_{\mathrm{B}}$ dopants in the 4-8 $\mathrm{THz}$ range, as defined in Eq. (1).

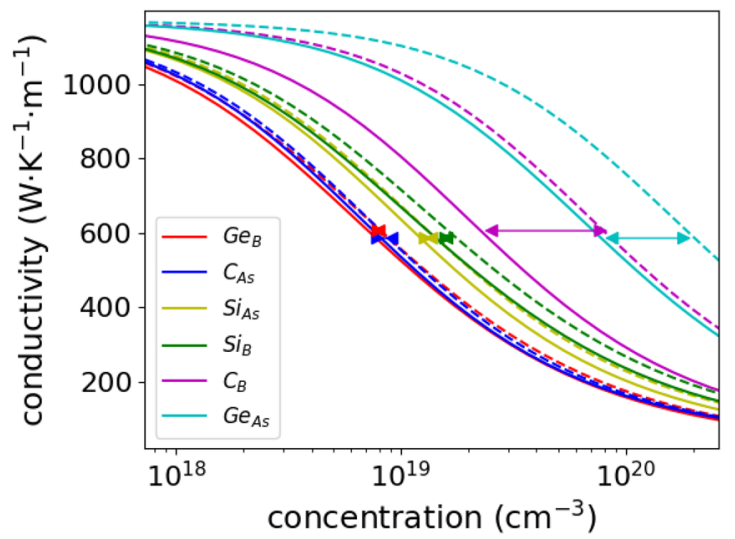

Fig. 3 BAs thermal conductivity vs. doping concentration. Lattice thermal conductivity of BAs vs doping concentration at $300 \mathrm{~K}$ for each of the considered impurities. Solid lines: neutral impurities. Dotted lines: charged impurities. Colored arrows indicate the difference between charged and neutral impurity concentrations that produce a $50 \%$ reduction in the BAs $K$.

densities achieved for each of the charged impurities compared with their neutral counterparts highlight the weaker scattering of phonons for impurities in their charged states. The $C_{A s}$ and $\mathrm{Ge}_{B}$ defects suppress the thermal conductivity the most, with a $50 \%$ reduction to $\sim 600 \mathrm{~W} \cdot \mathrm{m}^{-1} \cdot \mathrm{K}^{-1}$ at a defect concentration of $\sim 10^{19}$ $\mathrm{cm}^{-3} ; \mathrm{Si}_{\text {As }}$ closely follows, because its large mass variance dominates over the otherwise weak bond defect scattering. Since $\mathrm{Si}$ and $\mathrm{C}$ are known contaminants in BAs growth ${ }^{11}$, the present finding that they strongly reduce the BAs $K$ motivates synthesis approaches that minimize their presence, if maximum thermal conductivity is desired. Interestingly, $\mathrm{Si}_{\mathrm{B}}$ does provide quite a large reduction of $\kappa$ in both the neutral and charged cases despite having relatively weak low frequency mass defect scattering rates, because the scattering induced by the bond perturbation is quite high between 4 and $8 \mathrm{THz}$, similar to both $\mathrm{C}_{\mathrm{As}}$ and $\mathrm{Ge}_{\mathrm{B}}$. This effect clearly cannot be captured by a pure mass defect perturbation. The opposite holds for $\mathrm{Ge}_{\mathrm{As}}$ : it gives the smallest reduction to the BAs thermal conductivity for both charged and neutral cases, where phonon-impurity scattering rates are about an order of magnitude smaller than those for the $C_{A s}$ substitution in the critical $4-8 \mathrm{THz}$ range.
In the $\mathrm{C}_{\mathrm{As}}, \mathrm{Ge}_{\mathrm{B}}$ and $\mathrm{Si}_{\mathrm{As}}$ cases the mass variance hides the effect produced by the structural relaxation and by the change in the local bonds to such degree that the $\kappa$ differences between neutral and charged states are small, especially in the $\mathrm{Si}_{\mathrm{As}}$ case where the bond perturbation is already weak, see Fig. 3. Instead, the effect of $V_{\mathrm{K}}$ plays a much more relevant role when the mass variance is weak, that is in the $\mathrm{Si}_{B}, C_{B}$ and $\mathrm{Ge}_{A s}$ cases. In particular, in the latter two it constitutes the largest component of the perturbation and the difference between neutral and charged states is more marked. Moreover, for the $\mathrm{Si}_{\mathrm{B}}$ substitution, relatively weak rates at low frequency $(\sim<4 \mathrm{THz})$ in the charged case are compensated by a much stronger perturbation at high frequency.

\section{Effect of compensation}

In Fig. 3, each curve corresponds to the reduction of the BAs thermal conductivity assuming that the density of a particular impurity can be varied independently. We now consider a more complex behavior that might occur if the BAs growth process were governed by equilibrium thermodynamics. For low densities of the three dopants $\mathrm{D}=\mathrm{C}, \mathrm{Si}, \mathrm{Ge}$, the calculated formation energies for the charged acceptors, $\mathrm{Si}_{\mathrm{As}}^{-1}, \mathrm{Ge}_{\mathrm{As}}^{-1}$ and $\mathrm{C}_{\mathrm{As}}^{-1}$, are much lower than those for charged donors $^{11,12}$ so these impurities will form first. As the doping density increases and the Fermi level, $\epsilon_{\mathrm{F}}$, shifts towards the valence band edge, the D-acceptor formation energies increase while D-donor formation energies decrease ${ }^{11,12}$ thereby increasing the probability of D-donor formation. At the crossing point of the acceptor and donor formation energies, acceptors and donors form with equal probability and $\epsilon_{\mathrm{F}}$ becomes pinned. Thus, equilibrium growth thermodynamics mandates that beyond a certain concentration, adding more Dimpurities will form not only D-acceptors but also compensating Ddonors. In addition, temperature dependent mixtures of charged and neutral acceptors coexist because of the finite acceptor ionization energy. Therefore, understanding how donor compensation and the differences between neutral and charged defect scattering rates could shape dependence of the BAs thermal conductivity versus impurity concentration should be considered.

In the case of $\mathrm{Ge}$ doping, at lower densities $\mathrm{Ge}_{\mathrm{As}}^{-1}$ defects reduce $\kappa$ only slightly. But once $\mathrm{Ge}_{\mathrm{B}}^{+1}$ starts to form, $k$ decreases more rapidly due to the much larger scattering rates of the latter. The opposite behavior occurs for $C$ doping since $C_{A s}^{-1}$ scatters phonons more strongly than $\mathrm{C}_{\mathrm{B}}^{+1}$. The actual donor concentration depends on the impurity formation energies, on band structure-related quantities and on the experimental growth conditions as well - thus its precise ab initio calculation is challenging. Nevertheless, we employed the formation energy curves and ionization energies calculated from first principles by Chae et al. ${ }^{10}$, which gave the following Fermi level pinning values (eV) for As-rich (B-rich) conditions: C: 0.4 (0.15), Si: 0.15 $(-0.05)$, Ge: $0.25(0.0)$. We used these values along with averaged effective masses $m_{\mathrm{h}}^{*} \sim 0.56$ and $m_{\mathrm{e}}^{*} \sim 0.4$ from ref. ${ }^{11}$ to estimate the concentrations of charged and neutral acceptors and of compensating donors for each impurity density applying the charge neutrality condition at an assumed growth temperature of $1163 \mathrm{~K}$, consistent with measurements ${ }^{20}$. By including the scattering rates for charged and neutral acceptors and the compensating donors at the transport temperature of $300 \mathrm{~K}$ we have calculated the BAs $K$ vs. impurity concentration curves in Fig. 4. Solid lines show the Gedoping and C-doping cases for B-rich and As-rich growth conditions. For comparison, dashed curves in Fig. 4 reproduce the $\mathrm{Ge}_{\mathrm{As}}^{-1}$ and $\mathrm{Ge}_{\mathrm{As}}^{0}$ curves from Fig. 3. Including the effects of compensation hardly changes the curves for Si compared with those already plotted in Fig. 3 so they are omitted from Fig. 4. The change of dependence in the case of Ge doping is particularly evident. When grown in B-rich conditions, BAs could in principle be p-doped with Ge to nearly $10^{19} \mathrm{~cm}^{-3}$, without much decrease in the thermal conductivity, with somewhat smaller but still high $\kappa$ values achieved for the As-rich case. Beyond this doping level, the thermal conductivity would decrease more rapidly compared with the $\mathrm{Ge}_{\mathrm{As}}^{-1}$ - only case, with 


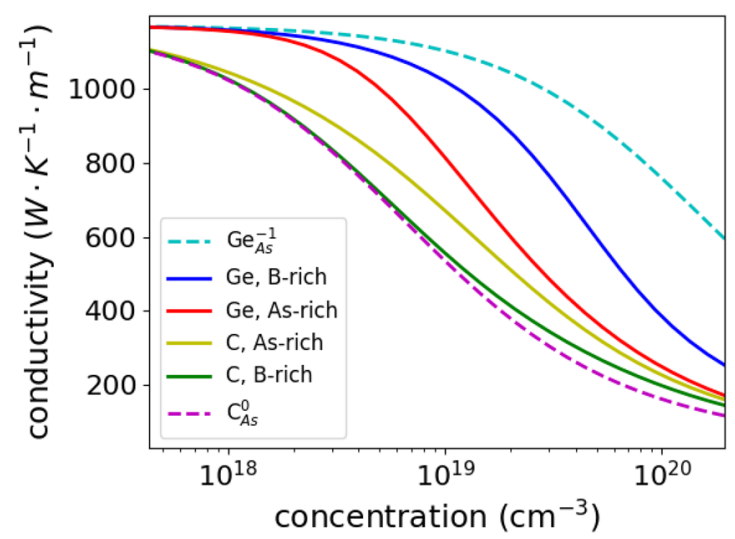

Fig. 4 Effect of donor compensation on BAs thermal conductivity. Lattice thermal conductivity of BAs vs Ge and $C$ impurity concentration at $300 \mathrm{~K}$ including effects of donor compensation. Results for both As-rich and B-rich conditions are given. Dashed curves $\mathrm{Ge}_{A s}^{-1}$ and $\mathrm{C}_{\mathrm{As}}^{0}$ are taken from Fig. 3 and included for comparison.

detrimental consequences for device heating. In contrast to the Ge doping, increased C-doping density leads to the opposite behavior. Since phonon scattering from $C_{B}^{+1}$ is very weak compared with that from $C_{A s}^{-1}$, the $C$ curves including compensation in Fig. 4 lie above the $C_{A s}$ curve that neglects compensation. The difference is particularly noticeable for the case of B-rich growth. We note that As-rich conditions are known to be more favorable for growth, whereas growth of B-rich BAs can be hampered by formation of the subarsenide phase $\mathrm{B}_{6} \mathrm{As}^{11}$.

\section{DISCUSSION}

With increasing Si, Ge and $\mathrm{C}$ impurity densities, free hole densities first increase, then saturate and finally decrease due to compensation from electrons ionized from increasingly large concentrations of donor atoms, as shown in Supplementary Fig. 2. Maximum hole densities of $2 \times 10^{18} \mathrm{~cm}^{-3}, 1 \times 10^{18} \mathrm{~cm}^{-3}$, and $1.5 \times 10^{17} \mathrm{~cm}^{-3}$ are obtained for $\mathrm{Si}, \mathrm{Ge}$ and $\mathrm{C}$, respectively. The lower value for $\mathrm{C}$ results primarily from its larger acceptor ionization of $0.09 \mathrm{eV}$ compared with the corresponding value of $0.03 \mathrm{eV}$ for both $\mathrm{Ge}$ and $\mathrm{Si}$, as calculated $a b$ initio ${ }^{10}$. These results are for impurity formation energies for the As-rich growth conditions expected to apply in current BAs synthesis. Ignoring donor compensation (dashed curves in Supplementary Fig. 2) gives similar results for $\mathrm{Si}$ and Ge up to impurity densities of around $10^{19} \mathrm{~cm}^{-3}$. These finding suggest that there is not much gain in hole density for impurity concentrations much above a few times $10^{18} \mathrm{~cm}^{-3}$, especially when high thermal conductivity is desired.

For the experimentally achievable As-rich growth, Ge doping shows a clear advantage over $\mathrm{C}$ and $\mathrm{Si}$ doping as donor compensation starts to be important only at concentrations approaching $10^{19} \mathrm{~cm}^{-3}$. More broadly, for all three dopants, the BAs $\mathrm{k}$ exceeds $600 \mathrm{~W} \cdot \mathrm{m}^{-1} \cdot \mathrm{K}^{-1}$ even at high dopant densities, thus retaining values far above those of common semiconductors such as Si $\left(140 \mathrm{~W} \cdot \mathrm{m}^{-1} \cdot \mathrm{K}^{-1}\right)$ and GaAs $\left(45 \mathrm{~W} \cdot \mathrm{m}^{-1} \cdot \mathrm{K}^{-1}\right)$. This could be a great advantage in applications where efficient heat dissipation is crucial. Furthermore, the results in Fig. 4 suggest a complementary experimental way to determine if compensation doping is occurring by directly measuring the thermal conductivity. Further calculations and experiments may be envisaged in this way, to evaluate the effect of compensation on the thermal transport properties in semiconductors. Finally, we note that while charged $C_{B}$ substitutions in the absence of $C_{A s}$ would give only minimal reduction of the BAs $K$ up to high densities, use of $C$ as an n-type dopant would be hindered by the large $C_{B}$ formation and ionization energies ${ }^{10,11}$.
In conclusion, we have evaluated the thermal conductivity reduction induced by doping $B A s$ with $C$, Si and $G e . G_{A s}$ and $C_{B}$ substitutions give exceptionally small reductions to the $B A s$ thermal conductivity even at high densities. However, the large formation and ionization energies of $C_{B}$ donors hinder their utility as n-type dopants and motivates a search for other candidates. Both $\mathrm{Si}$ and $\mathrm{Ge}$ achieve reasonably high hole densities while retaining high $\mathrm{BAs}$ thermal conductivity. Importantly, even at high impurity densities the BAs $\kappa$ significantly exceeds those of common semiconductors, highlighting its potential as a nextgeneration self-cooling functional material. An observable drop (enhancement) in thermal conductivity with respect to the charged D-acceptor case is predicted and explained upon $\mathrm{Ge}(\mathrm{C})$ doping if we consider compensating donor scattering centers and the temperature/doping dependence for the acceptor activation. This imposes practical limitations to be considered when designing BAs - based devices. It also suggests a direct alternative way to experimentally determine if a sample suffers from compensation doping. Finally, we have computationally identified a general phenomenon whereby charged impurities isoelectronic with the substituted species scatter phonons noticeably more weakly than their corresponding neutral counterparts. This phenomenon deserves further investigation in other systems with thermodynamically stable neutral impurities.

\section{METHODS}

\section{BTE and thermal conductivity}

The phonon Boltzmann Tranport Equation (BTE) ${ }^{14,15}$ is solved for the nonequilibrium phonon distribution function resulting from an applied temperature gradient, $\nabla \mathbf{T}$. From this, the thermal conductivity can be obtained, as described below. For small $\nabla \mathbf{T}$, the BTE can be linearized and solved self-consistently using the single mode relaxation time approximation (SMRTA) as a starting guess. By keeping only the terms linear in $\nabla \mathbf{T}$, for a given mode $\lambda \equiv(\mathbf{q}, s)$ where $\mathbf{q}$ is a phonon wave-vector and $s$ is a phonon branch, one can obtain the deviation from equilibrium for its phonon distribution, i.e., $n_{\lambda}=n_{\lambda}^{0}-\partial_{T} n_{\lambda}^{0} \mathbf{F}_{\lambda} \cdot \boldsymbol{\nabla T}$, where $\mathbf{F}_{\lambda}=\tau_{\lambda}^{0}\left(\mathbf{v}_{\lambda}+\boldsymbol{\Delta}_{\lambda}\left[\mathbf{F}_{\lambda}\right]\right), \tau_{\lambda}^{0}$ is the phonon relaxation time, $\mathbf{v}_{\lambda}$ is the phonon group velocity, $n_{\lambda}^{0}$ is the Bose-Einstein distribution and $\boldsymbol{\Delta}$ is a linear functional of $\mathbf{F}_{\lambda}$. We can interpret $\mathbf{F}_{\lambda}$ as a vector mean free path that measures the deviation from equilibrium induced by the thermal gradient. $\Delta \equiv 0$ corresponds to the SMRTA. In this work we consider three-, four-, and defect-mediated twophonon scattering processes ${ }^{15,21}$, the latter involving either isotopes or substitutional impurities. Here only the three-phonon scattering is included in $\Delta_{\lambda}$ while the four-phonon and defect scattering is included at the SMRTA level only with an acceptable level of accuracy, as the former is dominated by Normal processes while the latter involve mostly Umklapp processes ${ }^{5}$. The phonon thermal conductivity tensor $(\alpha, \beta$ denote Cartesian directions) is:

$\kappa^{\alpha \beta}=\frac{k_{B}}{\Omega_{s}} \sum_{\lambda}\left(\frac{\hbar \omega_{\lambda}}{k_{B} T}\right)^{2} n_{\lambda}^{0}\left(n_{\lambda}^{0}+1\right) v_{\lambda}^{\alpha} F_{\lambda}^{\beta}$

where $k_{B}$ is the Boltzmann constant, $\Omega_{s}$ is the crystal volume and $\omega_{\lambda}$ is the phonon angular frequency. Due to the cubic symmetry of BAs, the diagonal elements of the tensor are identical, thus it is clearly meaningful to consider only $k=\Sigma_{a} k^{a \alpha} / 3$.

\section{Scattering rates and phonon-defect interaction}

The total relaxation time $\tau_{\lambda}^{0}$ can be computed from the Matthiessen's rule:

$\frac{1}{\tau_{\lambda}^{0}}=\frac{1}{\tau_{\lambda}^{3 p h}}+\frac{1}{\tau_{\lambda}^{4 p h}}+\frac{1}{\tau_{\lambda}^{\text {iso }}}+\frac{1}{\tau_{\lambda}^{\text {def }}}$

The non-harmonic three- and four-phonon scattering rates are evaluated from first principles by computing the third and fourth-order force constants ${ }^{3,15}$. We rely on the work of Tamura et al. ${ }^{22}$ to assess the phonon-isotope interaction, whilst a T-matrix based approach has been developed to treat the substitutional impurities that involve both a mass $V_{\mathrm{M}}$ and a bond perturbation $V_{\mathrm{K}}$ near the impurity ${ }^{16,17}$. The total 
perturbation is thus defined as $V=V_{\mathrm{M}}+V_{\mathrm{K}}$, where:

$$
V_{M}^{i j, a \beta}\left(\omega_{\lambda}\right)=-\omega_{\lambda}^{2} \frac{\tilde{M}_{i}-M_{j}}{M_{i}} \delta_{i j} \delta_{a \beta}
$$

and

$V_{\mathrm{K}}^{i j, a \beta}=\frac{\tilde{K}^{i j, a \beta}-K^{i j, a \beta}}{\sqrt{M_{i} M_{j}}}$

here $i, j$ identify atoms in the supercell; $M_{i}\left(\tilde{M}_{i}\right)$ is the mass of the host (substitution) atom at site $i$; and $K^{i j, a \beta}\left(\tilde{K}^{i j, a \beta}\right)$ is the harmonic force constant tensor element between atoms $i$ and $j$ in the pristine (defective) supercell. Once $V$ is known, one can calculate the pristine, retarded Green's function:

$\left\langle i a\left|G_{0}^{+}\left(\omega^{2}\right)\right| j \beta\right\rangle=\sum_{\lambda}\langle i a \mid \lambda\rangle\langle\lambda \mid j \beta\rangle\left(\omega^{2}-\omega_{\lambda}^{2}+i 0^{+}\right)^{-1}$

and finally the scattering T-matrix to all orders:

$T^{+}=\left(1-V G_{0}^{+}\right)^{-1} V$

We assume that the defects are randomly distributed throughout the crystal and that their concentration is sufficiently low that each impurity can be treated as an independent scattering center. Since the defect concentrations considered here are dilute, less than around $0.1 \%$ of either As or B atoms, effects associated with phonon scattering from multiple defects can be ignored ${ }^{23}$. At such low concentration we can also ignore any frequency renormalization induced by impurities, as shown in Supplementary Fig. 3. Thus, the phonon-defect scattering rates can be expressed as:

$\frac{1}{\tau_{\lambda}^{\text {ph-def }}}=-\chi \frac{V_{\mathrm{uc}}}{\omega_{\lambda}} \Im\left\langle\lambda\left|T^{+}\right| \lambda\right\rangle$

where $x$ is the volume concentration of impurities in the system and $V_{\mathrm{uc}}$ is the unit cell volume.

\section{Charged/neutral impurity densities and donor compensation}

With increasing impurity density, the magnitude of neutral and charged defects varies. Of particular importance: donor compensation begins to occur after a certain doping level is reached ${ }^{10}$. To account for this, we combine the effect of the neutral and ionized acceptors and the donors to a single rates expression using Matthiessen's rule. To estimate the concentrations of charged and neutral impurities and compensating donors formed during growth, we impose the charge neutrality condition, namely

$p-n=N_{\mathrm{A}}^{-}-N_{\mathrm{D}}^{+}$

where $p, n, N_{\mathrm{A}}^{-}$and $N_{\mathrm{D}}^{+}$are, respectively, the hole, electron, ionized acceptor and ionized donor concentrations. The standard expressions for $p$ and $n$ are:

$p\left(\epsilon_{\mathrm{F}}, T\right) \equiv \int_{-\infty}^{\epsilon_{\mathrm{VBM}}} D_{h}(\epsilon)\left(1-f\left(\epsilon ; \epsilon_{\mathrm{F}}, T\right)\right) d \epsilon$

$n\left(\epsilon_{\mathrm{F}}, T\right) \equiv \int_{\epsilon_{\mathrm{CBM}}}^{\infty} D_{e}(\epsilon) f\left(\epsilon ; \epsilon_{\mathrm{F}}, T\right) d \epsilon$

where $D_{h}(\epsilon)$ and $D_{e}(\epsilon)$ are the hole and electron Density of States (DOS), $\epsilon_{\mathrm{VBM}}$ and $\epsilon_{\mathrm{CBM}}$ are the Valence Band Maximum and Conduction Band Minimum, $\epsilon_{\mathrm{F}}$ is the Fermi Level and $f\left(\epsilon_{;} \epsilon_{\mathrm{F}}, T\right)$ is the equilibrium Fermi Dirac distribution. A simple isotropic parabolic band model has been employed to evaluate the hole- and electron- DOS near the gap:

$$
\begin{aligned}
& D_{h}(\epsilon)=\frac{1}{2 \pi^{2}}\left(\frac{2 m_{\mathrm{h}}^{*}}{\hbar^{2}}\right)^{3 / 2} \sqrt{\epsilon_{\mathrm{VBM}}-\epsilon} \\
& D_{e}(\epsilon)=\frac{1}{2 \pi^{2}}\left(\frac{2 m_{\mathrm{e}}^{*}}{\hbar^{2}}\right)^{3 / 2} \sqrt{\epsilon-\epsilon_{\mathrm{CBM}}}
\end{aligned}
$$

Here, $\epsilon_{\mathrm{CBM}}$ and $\epsilon_{\mathrm{VBM}}$ are the electronic energies at the conduction and valence band edges. Now, given the acceptor and donor electronic levels $\epsilon_{\mathrm{A}}$ and $\epsilon_{\mathrm{D}}$ we consider

$N_{\mathrm{A}}^{-}=\frac{N_{\mathrm{A}}}{1+4 \cdot \exp \left(\left(\epsilon_{\mathrm{A}}-\epsilon_{\mathrm{F}}\right) / k_{\mathrm{B}} T\right)} \equiv f_{\mathrm{A}} \cdot N_{\mathrm{A}}$ and

$$
N_{\mathrm{D}}^{+}=\frac{N_{\mathrm{D}}}{1+2 \cdot \exp \left(\left(\epsilon_{\mathrm{F}}-\epsilon_{\mathrm{D}}\right) / k_{\mathrm{B}} T\right)} \equiv f_{\mathrm{D}} \cdot N_{\mathrm{D}}
$$

along with $N_{\mathrm{A}}+N_{\mathrm{D}}=X$, where $X$ is the total concentration of impurities, and we define $g \equiv N_{D} / N_{A}$. The value of $f_{D}$ can be safely assumed to be equal to one, since $\left(\epsilon_{\mathrm{D}}-\epsilon_{\mathrm{F}}\right) \gg k_{B} T$ for all the considered doping range. Therefore we have $N_{\mathrm{A}}=\chi /(1+g)$ and $N_{\mathrm{D}}^{+} \simeq N_{\mathrm{D}}=g X /(1+g)$.

The definition of $g$ is of paramount importance for the determination of the fraction of donors and acceptors. Here we have assumed that impurities are cast into BAs at growth - temperature $T_{\text {growth }}=1163 \mathrm{~K}^{20}$ and that diffusion processes are negligible, which results in fixed $N_{\mathrm{A}}$ and $N_{\mathrm{D}}$ when the temperature is lowered. To do that, we have first solved Eq. (9) by taking $g$ at $T_{\text {growth }}$ with $N_{\mathrm{D}}^{+} / N_{\mathrm{A}}^{-}=\exp \left[-\left(E_{\mathrm{D}}-E_{\mathrm{A}}\right) /\left(k_{B} T_{\text {growth }}\right)\right]$, where $E_{A}$ and $E_{D}$ are respectively the formation energies for the charged acceptor and charged donor state ${ }^{10,11}$. The expression for $g$ stems from the Gibbs free energy for the doped system, where the contribution from vibrational entropy has been neglected. The ensuing and fixed values of $N_{A}\left(T_{\text {growth }}\right)$ and $N_{D}\left(T_{\text {growth }}\right)$ have been utilized for each value of $X$ to evaluate $f_{A}$ at $T=$ $300 \mathrm{~K}$. Finally, if we set $V_{\mathrm{uc}} \Im\left\langle T_{\mathrm{DEF}}^{+}\right\rangle_{\lambda} / \omega_{\lambda} \equiv \mathrm{B}_{\lambda}^{\mathrm{DEF}}$ for each type and state of defect, we have:

$\frac{1}{\tau_{\lambda, \text { tot }}^{\text {def }}(X)}=N_{A}^{-} B_{\lambda}^{\text {ch.,acc. }}+N_{A}^{0} B_{\lambda}^{\text {ne.,acc. }}+N_{D}^{+} B_{\lambda}^{\text {ch., don. }}$

\section{Computational details}

We use the VASP ${ }^{24-26}$ DFT code employing the PAW ${ }^{27,28}$ pseudopotentials in the $\mathrm{PBE}^{29,30}$ approximation for the phonon calculations. First, the second order force constants (IFC2) are calculated for a relaxed $5 \times 5 \times 5(250$ atom) supercell with and without a substitution defect, using the small displacement method. Phonopy package $e^{31,32}$ is used to create the supercells with small displacements, and to read off force constants following DFT calculations. To calculate $V_{\mathrm{K}}$, we first proceeded by taking the difference between the IFC2s of the pristine and the defective system.

Whereas our procedure is implemented in real space, to reduce the computational workload we consider the local nature of the bond perturbation and we choose two cutoffs, namely $r_{\text {cut }}$ and $R_{\text {cut. }}$ One cutoff represents the maximum distance that allows interactions between pairs of atoms, at least one of which belongs to the list of neighbors of the defect site selected by $R_{\text {cut }}$. After convergence tests we have chosen the pair- and the neighbor list- cutoffs as $0.6-0.8 \mathrm{~nm}$ respectively. Once these values are defined, it is necessary to reinforce the acoustic sum rule: this is done by projecting away the degrees of freedom corresponding to rigid translations ${ }^{33}$. Despite the insulating nature of BAs, we can observe that the character of the state when introducing neutral impurities is metallic. In this case we must use a certain care when evaluating the interatomic forces and force constants: if the VASP smearing parameters were chosen to be as for insulators, this would lead to long range interactions and thus absence of convergence for the phonon-defect scattering rates with respect to the cutoffs. In this work we used the default VASP smearing parameters (ISMEAR $=1$, SIGMA $=0.2$ ), comparing with volume-relaxation and spin-relaxation (non-collinear) calculations as well and finding that the latter do not affect rates and thermal conductivity. A converged value for $r_{\text {cut }}$ and $R_{\text {cut }}$ was found in all the calculations. To account for the charged impurity states, we raise (As site substitutions) or lower (B site substitutions) the total number of valence electrons for the substituted atom by 1 with respect to the neutral impurity case and then a compensating uniform positive or negative background charge is added to the supercell to maintain charge neutrality.

The scattering T-matrix is then calculated using $V=V_{\mathrm{K}}+V_{\mathrm{M}}$ and the Green's function using our home-grown code. Once the T-matrix is known, the phonon-substitution defect scattering rates are calculated for various defect concentrations. These are explicitly shown in Supplementary Figs. $4-9$ at the concentration of $3.26 \cdot 10^{18} \mathrm{~cm}^{-3}$, considering the mass-only, bond-only and total perturbations in both the charged and neutral state and for all the considered impurities. To calculate the three-phonon scattering rates, we use the thirdorder vasp.py code ${ }^{34}$ in conjunction with VASP and almaBTE ${ }^{35}$. The calculation of the four-phonon scattering rates are expensive. Moreover, our home-grown four-phonon code is currently set up to work only with the Quantum Espresso ${ }^{36}$ suite. We simply interpolate these scattering rate on the wave vector mesh used in the current study from a previously published calculation ${ }^{3}$. The phonon-substitution defect scattering rates are combined with the threeand four-phonon, and phonon-isotope scattering rates at the relaxation 
time approximation level using Matthiesen's rule inside the almaBTE code. almaBTE finds the full solution of the linearized phonon BTE, and outputs the thermal conductivity $\kappa$. A converged $28 \times 28 \times 28$ transport wave vector mesh is used to solve the phonon BTE, whilst the Green's function is evaluated on a $16 \times 16 \times 16$ grid. Values for the $\mathrm{Si}, \mathrm{C}$, and $\mathrm{Ge}$ acceptor and donor formation energies, $E_{\mathrm{A}}$ and $E_{\mathrm{D}}$, acceptor and donor ionization energies, $\epsilon_{\mathrm{A}}-\epsilon_{\mathrm{VBM}}$, and $\epsilon_{\mathrm{CBM}}-\epsilon_{\mathrm{D}}$ and energy gap have been taken from ref. ${ }^{10}$.

\section{DATA AVAILABILITY}

Four-phonon scattering rates used in the thermal conductivity calculations for BAs and VASP related files are available upon reasonable request. All other data is available from Zenodo at https://doi.org/10.5281/zenodo.4453192.

\section{CODE AVAILABILITY}

All formulations and algorithms necessary to reproduce the results of this study are described in the Methods section and in Refs. ${ }^{3}$ and ${ }^{35}$.

Received: 23 February 2021; Accepted: 11 March 2021; Published online: 19 April 2021

\section{REFERENCES}

1. Kang, J. S., Li, M., Wu, H., Nguyen, H. \& Hu, Y. Experimental observation of high thermal conductivity in boron arsenide. Science 361, 575-578 (2018).

2. Li, S. et al. High thermal conductivity in cubic boron arsenide crystals. Science 361, 579-581 (2018).

3. Tian, F. et al. Unusual high thermal conductivity in boron arsenide bulk crystals. Science 361, 582-585 (2018)

4. Lindsay, L., Broido, D. A. \& Reinecke, T. L. First-principles determination of ultrahigh thermal conductivity of boron arsenide: a competitor for diamond? Phys. Rev. Lett. 111, 025901 (2013).

5. Feng, T., Lindsay, L. \& Ruan, X. Four-phonon scattering significantly reduces intrinsic thermal conductivity of solids. Phys. Rev. B 96, 161201 (2017).

6. Liu, T.-H. et al. Simultaneously high electron and hole mobilities in cubic boron-V compounds: BP, BAs, and BSb. Phys. Rev. B 98, 081203 (2018).

7. Kim, J. et al. Thermal and thermoelectric transport measurements of an individual boron arsenide microstructure. Appl. Phys. Lett. 108, 201905 (2016).

8. Lv, B. et al. Experimental study of the proposed super-thermal-conductor: BAs. Appl. Phys. Lett. 106, 074105 (2015).

9. Zheng, Q. et al. Antisite pairs suppress the thermal conductivity of BAs. Phys. Rev. Lett. 121, 105901 (2018).

10. Chae, S., Mengle, K., Heron, J. T. \& Kioupakis, E. Point defects and dopants of boron arsenide from first-principles calculations: donor compensation and doping asymmetry. Appl. Phys. Lett. 113, 212101 (2018).

11. Lyons, J. L. et al. Impurity-derived p-type conductivity in cubic boron arsenide. Appl. Phys. Lett. 113, 251902 (2018).

12. Bushick, K., Mengle, K., Sanders, N. \& Kioupakis, E. Band structure and carrier effective masses of boron arsenide: effects of quasiparticle and spin-orbit coupling corrections. Appl. Phys. Lett. 114, 022101 (2019).

13. Ziman, J. Electrons and phonons: the theory of transport phenomena in solids. International series of monographs on physics (OUP Oxford, 2001).

14. Omini, M. \& Sparavigna, A. Beyond the isotropic-model approximation in the theory of thermal conductivity. Phys. Rev. B 53, 9064-9073 (1996).

15. Li, W., Carrete, J., Katcho, N. A. \& Mingo, N. ShengBTE: a solver of the Boltzmann transport equation for phonons. Comp. Phys. Commun. 185, 1747-1758 (2014).

16. Mingo, N., Esfarjani, K., Broido, D. A. \& Stewart, D. A. Cluster scattering effects on phonon conduction in graphene. Phys. Rev. B 81, 045408 (2010).

17. Economou, E. N. Green's functions in quantum physics (Springer, 2006). URL https://books.google.fr/books?id=s0gsAAAAYAAJ.

18. Lindsay, L., Broido, D. A. \& Reinecke, T. L. Phonon-isotope scattering and thermal conductivity in materials with a large isotope effect: a first-principles study. Phys. Rev. B 88, 144306 (2013).

19. Chen, K. et al. Ultrahigh thermal conductivity in isotope-enriched cubic boron nitride. Science 367, 555-559 (2020).

20. Tian, F. et al. Seeded growth of boron arsenide single crystals with high thermal conductivity. Appl. Phys. Lett. 112, 031903 (2018).

21. Feng, T. \& Ruan, X. Quantum mechanical prediction of four-phonon scattering rates and reduced thermal conductivity of solids. Phys. Rev. B 93, 045202 (2016).
22. Tamura, S.-i Isotope scattering of large-wave-vector phonons in GaAs and InSb: deformation-dipole and overlap-shell models. Phys. Rev. B 30, 849-854 (1984).

23. Walton, D. Phonon-defect interaction. (Springer US: Boston, MA, 1975) 393-440.

24. Kresse, G. \& Hafner, J. Ab initio molecular dynamics for liquid metals. Phys. Rev. $B$ 47, 558 (1993).

25. Kresse, G. \& Hafner, J. Ab initio molecular-dynamics simulation of the liquidmetal-amorphous-semiconductor transition in germanium. Phys. Rev. B 49, 14251 (1994).

26. Kresse, G. \& Furthmüller, J. Efficient iterative schemes for ab initio total-energy calculations using a plane-wave basis set. Phys. Rev. B 54, 11169 (1996).

27. Blöchl, P. E. Projector augmented-wave method. Phys. Rev. B 50, 17953 (1994).

28. Kresse, G. \& Joubert, D. From ultrasoft pseudopotentials to the projector augmented-wave method. Phys. Rev. B 59, 1758 (1999).

29. Perdew, J. P., Burke, K. \& Ernzerhof, M. Generalized gradient approximation made simple. Phys. Rev. Lett. 77, 3865 (1996).

30. Burke, K., Perdew, J. P. \& Ernzerhof, M. Why semilocal functionals work: accuracy of the on-top pair density and importance of system averaging. J. Chem. Phys. 109, 3760-3771 (1998).

31. Togo, A. \& Tanaka, I. First principles phonon calculations in materials science. Scr. Mater. 108, 1-5 (2015).

32. Togo, A., Oba, F. \& Tanaka, I. First-principles calculations of the ferroelastic transition between rutile-type and $\mathrm{CaCl}_{2}$-type $\mathrm{SiO}_{2}$ at high pressures. Phys. Rev. $B$ 78, 134106-134114 (2008).

33. Katre, A., Carrete, J., Dongre, B., Madsen, G. K. H. \& Mingo, N. Exceptionally strong phonon scattering by B substitution in cubic SiC. Phys. Rev. Lett. 119, 075902 (2017).

34. Li, W., Lindsay, L., Broido, D. A., Stewart, D. A. \& Mingo, N. Thermal conductivity of bulk and nanowire $\mathrm{Mg}_{2} \mathrm{Si}_{x} \mathrm{Sn}_{1-x}$ alloys from first principles. Phys. Rev. B 86, 174307 (2012).

35. Carrete, J. et al. almaBTE: a solver of the space-time dependent Boltzmann transport equation for phonons in structured materials. Comput. Phys. Commun. 220, 351-362 (2017).

36. Giannozzi, P. et al. QUANTUM ESPRESSO: a modular and open-source software project for quantum simulations of materials. J. Phys. Condens. Matter 21, 395502 (2009).

\section{ACKNOWLEDGEMENTS}

This work was supported in part by the Office of Naval Research under MURI grant no. N00014-16-1-2436, and the Agence Nationale de la Recherche through project ANR-17-CE08-0044-01. G.K.H.M. acknowledges funding from the Austrian Science Funds (FWF) under project CODIS (Grant no. FWF-I-3576-N36). We thank Nebil Katcho for providing us with the first version of the code used to compute the phonondefect scattering rates. D.B. thanks Dr. John Lyons of the Naval Research Laboratory for helpful discussions.

\section{AUTHOR CONTRIBUTIONS}

M.F. and N.H.P. contributed equally. M.F., N.H.P. and C.L. performed the first-principles calculations. M.F., N.H.P. N.M. and D.B. wrote the manuscript and developed the analytical modeling. N.M. and D.B. supervised the research. All authors analyzed the data and commented on, discussed and edited the manuscript.

\section{COMPETING INTERESTS}

The authors declare no competing interests.

\section{ADDITIONAL INFORMATION}

Supplementary information The online version contains supplementary material available at https://doi.org/10.1038/s41524-021-00519-3.

Correspondence and requests for materials should be addressed to D.B.

Reprints and permission information is available at http://www.nature.com/ reprints

Publisher's note Springer Nature remains neutral with regard to jurisdictional claims in published maps and institutional affiliations. 
Open Access This article is licensed under a Creative Commons Ac Attribution 4.0 International License, which permits use, sharing,
adaptation, distribution and reproduction in any medium or format, as long as you give appropriate credit to the original author(s) and the source, provide a link to the Creative Commons license, and indicate if changes were made. The images or other third party material in this article are included in the article's Creative Commons license, unless indicated otherwise in a credit line to the material. If material is not included in the article's Creative Commons license and your intended use is not permitted by statutory regulation or exceeds the permitted use, you will need to obtain permission directly from the copyright holder. To view a copy of this license, visit http://creativecommons. org/licenses/by/4.0/.

(c) The Author(s) 2021 